\title{
Ghrelin as a novel peptide hormone in obesity and weight loss
}

\author{
W. Liu ${ }^{1}$, M. Xiang ${ }^{2}$, L. S. Harbige ${ }^{2}$ and H. Ai ${ }^{1}$ \\ ${ }^{1}$ Division of Nutrition and Biochemistry, Institute of Sports Medicine, The Third Hospital, Peking University, Beijing, \\ P.R. China and ${ }^{2}$ Centre for Biosciences Research, School of Science, University of Greenwich, Kent ME4 4TB, UK
}

Obesity is associated with an increased risk of disease such as cardiovascular disease, hypertension and type 2 diabetes ${ }^{(1)}$. It is estimated that overweight and obesity in the UK contributes to more than 65000 deaths and around $5 \%$ of total NHS expenditure annually ${ }^{(2)}$. Ghrelin which is predominantly produced by the stomach, is regarded as a novel peptide hormone and a potent stimulator of growth hormone release, food intake and weight gain ${ }^{(3)}$. The model of diet-induced obesity (DIO) in rats has many features in common with human obesity and can serve as a useful model to study the pathogenesis and treatment of obesity. The present study determined the effect of weight loss on plasma ghrelin and expression of gastric ghrelin gene in DIO and DIO-restricted rats. Male Sprague-Dawley rats $(n=110)$ at 3 weeks of age were randomly assigned into groups. Ten rats were fed rat chow during the study as the control and 100 rats were fed both rat chow plus high fat diet [HFD, $5.58 \mathrm{kcal} / \mathrm{g}(23.35 \mathrm{~kJ} / \mathrm{g})$ with $66.5 \%$ as fat $/$ lard] ad libitum for 14 weeks. At the end of this period, 44 rats developed DIO which was assessed by the Lee obesity index ${ }^{4}$ ). The DIO rat's Lee obesity index was more than the average Lee obesity index plus 2 SD of the chow-fed control rats. At week 15, 10 chow-fed control rats were kept on the rat chow, 11 DIO rats were kept on the rat chow plus HFD ad libitum and the other 11 DIO rats that were designated as DIO-restricted, were restricted on the intake of the rat chow plus HFD for 5 weeks. Other rats were used in other studies (data not presented). It was found that the body weight of DIO-restricted rats after the intake was restricted for 5 weeks was significantly less than that of DIO-restricted rats before the intake was restricted and that of DIO rats, however, the body weight of DIO-restricted rats after the intake restriction was significantly more than that of chow-fed control rats. DIO-restricted rats had significantly lower perirenal, epididymal and total fat pad weight than DIO rats, but, had significantly higher fat pad weight than chow-fed control rats. The plasma ghrelin concentration in DIO-restricted rats tended to be higher than that of DIO rats (Figure). Furthermore, the gene expression of gastric ghrelin in DIO-restricted rats was significantly higher than that of DIO rats and chow-fed control rats (Figure). The body weight and the abdominal fat depots weight were negatively correlated with the levels of plasma ghrelin and gene expression of gastric ghrelin. These results indicate that the elevated expression of ghrelin gene during weight loss may be the physiological basis of resistance to weight loss in obesity. Inhibiting ghrelin signaling therefore appears an attractive target for anti-obesity therapies.

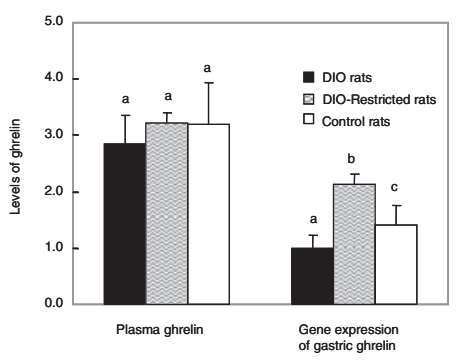

a, b, c, Mean values with unlike superscripts were significantly different $(P<0.05$ or $P<0.01)$.

1. Xiao H, Xiang M, Harbige LS \& Ai H (2010) Proc Nutr Soc 69, E324.

2. Allender S \& Rayner M (2007) Obes Rev 8, 467-473.

3. Ledderose C, Kreth S \& Beiras-Fernandez A (2011) Recent Pat Endocr Metab Immune Drug Discov 5, 1-6.

4. Bernardis LL \& Patterson BD (1968) J Endocrinol 40, 527-528. 